\title{
Resect and discard: Is it ready or time to shift gear?
}

\section{(ㄷ)(1) $(9)$}

\author{
Authors \\ Raf Bisschops ${ }^{1}$, Mário Dinis-Ribeiro² \\ Institutions \\ 1 University Hospital Gasthuisberg, Gastroenterology, \\ Belgium \\ 2 Instituto Português de Oncologia, Gastrenterologia, \\ Portugal
}

Bibliography

DOI https://doi.org/10.1055/a-1178-9711 |

Endoscopy International Open 2020; 08: E924-E926

\author{
(c) Georg Thieme Verlag KG Stuttgart · New York \\ eISSN 2196-9736 \\ Corresponding author \\ Prof. Mário Dinis-Ribeiro, Instituto Português de Oncologia, \\ Gastrenterologia, Rua Dr. Bernardino de Almedia, Porto, \\ 4200-072, Portugal \\ Fax: +351225504088 \\ mario@med.up.pt
}

Numerous colonoscopies are performed in Europe, either within organized colorectal cancer screening programs based on fecal immunochemical testing (FIT) or as primary screening procedures. In this process, accidental diminutive polyps are found that do not explain the positive FIT test and that may not significantly affect the cancer risk for that individual [1].

The resect and discard strategy constitutes an alternative to classical histopathology for small polyps that are the most common type found during colonoscopy. Microscopic analysis of these polyps, which have an intrinsic low risk of harboring advanced pathology, is very costly. As a consequence, real-time optical diagnosis with virtual chromoendoscopy entails a significant cost-saving potential for daily colorectal cancer screening and surveillance. Obviously, correctly differentiating between an adenoma and a hyperplastic polyp is crucial to implementing this strategy and to assessing the appropriate surveillance interval [2]. More than 10 years have passed since the feasibility and possible cost effectiveness of a resect and discard strategy was first demonstrated and suggested to be ready for prime time [3].

Recent European Society of Gastrointestinal Endoscopy (ESGE) guidelines for advanced imaging techniques incorporated this strategy as a valid option to replace histology under strict conditions of using validated scales, training and auditing of the practice [4]. Advanced imaging techniques like narrow band imaging (NBI) and classical chromoendoscopy have been around for more than 15 years now. We have validated scales like the Workgroup serrated polypS and Polyposis (WASP) classification, NBI International Colorectal Endoscopic (NICE) classification and more recently the BLI Adenoma Serrated International (BASIC) classification and simplified endoscopic classification to predict polyp histology (SIMPLE) classification for blue light imaging and I-scan that show high diagnostic accuracy [5-9].
So what takes us so long to go ahead, shift gear, and start applying all this knowledge and evidence in practice? Why are we still wasting millions of euros on the microscopic analysis of clinically insignificant polyps?

There are three reasons for this hesitance and cold water fear. First, there is the evidence sprouting from more real-life settings in daily endoscopy practices. Indeed the DISCARD II trial conducted in 1688 patients in routine clinical practice showed an insufficient test sensitivity of NBI optical diagnosis for diagnosing adenomas and predicting the correct surveillance interval [10]. Recently, a Dutch multicenter study looked at the performance of optical diagnosis in the Dutch colorectal screening program and found a too-low specificity of approximately $50 \%$ for adenomas and accuracy between $70 \%$ and $79 \%$ [11]. The concerns and uncertainties resulting from these studies lead us to the second barrier for implementation. The endoscopist is not convinced that he or she can do this. This is nicely demonstrated by the findings of a large international survey published in this issue of EIO [12]. Willems et al conducted a survey with 808 endoscopists, mainly practicing in northern America, who answered questions addressing their current use of the resect and discard strategy. Eight-four percent of the endoscopists are not using this strategy and more importantly, $60 \%$ believe that it is not feasible to implement in its current form. The authors showed clear geographical differences with application of a resect and discard strategy: in Europe by $39 \%$ of replicants, in Asia by $45 \%$, but in Canada and the United States only $13 \%$ and $5 \%$. In this survey, barriers for implementation were also noticeable: fear of making the wrong diagnosis (45\%), assigning the wrong interval (58\%) and also importantly, fear of medicolegal issues (54\%). A third potential barrier for implementation lies in the patient. Rex et al conducted a survey among American patients (corresponding to most of the respondents from the Willems survey) and found that the rate of 
acceptance by patients was only $66 \%$. In particular, y $50 \%$ of patients unwilling to accept a resect and discard strategy wanted an absolute zero chance of cancer in diminutive polyps and were willing to pay out of their own pocket for histological assessment of these small polyps [13].

So indeed, if we put these three barriers together, it is too early to shift into a higher gear and have widespread implementation of resect and discard. Interestingly, the survey by Willems et al [12] also showed that although the majority of endoscopists do not believe that optical diagnosis can replace histology, $63 \%$ agreed that diminutive polyps can be left unresected until the next screening colonoscopy because of the low risk of cancer, but without a consensus on the correct follow-up after leaving those in place. Moreover, although the majority of endoscopists in North America were uncertain about making an optical diagnosis and implementing a resect and discard strategy, $55 \%$ of them admitted to leaving diminutive polyps when they appeared to be non-adenomatous. This apparent contradiction, however, entails a certain risk, because many of these endoscopists were never trained in optical diagnosis but nonetheless apparently use it. This indicates that although there is a certain hesitance, there is also a need for proper and correct implementation of optical diagnosis.

There are two possible ways to overcome this contradiction and the barriers.

The first one is a dedicated training program to implement optical diagnosis in a structured way. ESGE is currently finalizing a postgraduate curriculum for optical diagnosis throughout the gastrointestinal tract. The emphasis will be on the use of standardized training modules, feedback, and audit of practices both during and after training [14]. The fact that endoscopists will be able to follow a standardized training track and can show their diagnostic accuracy for optical diagnosis should facilitate implementation. Endoscopists will feel more assured and their patient can be convinced by the record of an endoscopist's training and performance. The latter should also help to deal with potential medico-legal issues; medicine is not an absolute science and we accept a $5 \%$ error margin in everyday diagnostic testing, such as with standard blood tests.

The second possible solution and probably the one that holds the biggest promise is automated diagnosis through artificial intelligence. In recent years, deep learning has revolutionized the field of computer-aided analysis and has also entered the medical world, with results matching or even surpassing human-expert-level performance [15]. For colorectal polyp detection, several pilot studies introducing automated systems for polyp segmentation and characterization have recently been published, but clinical validation in a real-life setting remains to be established [16-19]. There is a definite need to develop a system applicable to different endoscopy systems and that can be validated in a real-life clinical setting. Recently a system with that potential has been commercialized but has up to now not incorporated a module for optical diagnosis of polyps [20]. Systems that have the possibility for characterization have promising diagnostic performance and seem to outperform endoscopists, but the results still need to be confirmed in real-life clinical trials [21,22].
So, in conclusion, it is time to switch to second gear for optical diagnosis, that being proper training and subsequent implementation. Meanwhile, we await further validation of new Albased techniques that will pave the path for shifting to a sport modus with easier implementation. Results with performance of systems that are largely operator-independent and assessed in well-designed prospective trials will most likely be attractive to both endoscopists and patients. Optical diagnosis will eventually be like the lab tests that are performed daily, which once validated are also acceptable from a medico-legal point of view, which is one of the largest barriers identified in the survey by Willems et al.

\section{Competing interests}

The authors declare that they have no conflict of interest.

\section{References}

[1] Lieberman D, Moravec M, Holub J et al. Polyp size and advanced histology in patients undergoing colonoscopy screening: implications for CT colonography. Gastroenterology 2008; 135: 1100-1105

[2] Hassan C, Quintero E, Dumonceau J-M et al. Post-polypectomy colonoscopy surveillance: European Society of Gastrointestinal Endoscopy (ESGE) Guideline. Endoscopy 2013; 45: 842-864

[3] Ignjatovic A, East JE, Suzuki N et al. Optical diagnosis of small colorectal polyps at routine colonoscopy (Detect InSpect ChAracterise Resect and Discard; DISCARD trial): a prospective cohort study. Lancet Oncol 2009; 10: 1171-1178

[4] Bisschops R, East JE, Hassan C et al. Advanced imaging for detection and differentiation of colorectal neoplasia: European Society of Gastrointestinal Endoscopy (ESGE) Guideline - Update 2019. Endoscopy 2019; 51: 1155-1179

[5] ljspeert JEG, Bastiaansen BA], van Leerdam ME et al. Development and validation of the WASP classification system for optical diagnosis of adenomas, hyperplastic polyps and sessile serrated adenomas/ polyps. Gut 2016; 65: 963-970

[6] Hewett DG, Kaltenbach T, Sano Y et al. Validation of a simple classification system for endoscopic diagnosis of small colorectal polyps using narrow-band imaging. Gastroenterology 2012; 143: 599-607. e1

[7] Bisschops R, Hassan C, Bhandari P et al. BASIC (BLI Adenoma Serrated International Classification) classification for colorectal polyp characterization with blue light imaging. Endoscopy 2018; 50: 211-220

[8] Hassan C, Bisschops R, Bhandari P et al. Predictive rules for optical diagnosis of $10-\mathrm{mm}$ colorectal polyps based on a dedicated software. Endoscopy 2020; 52: 52-60

[9] lacucci M, Trovato C, Daperno M et al. Development and validation of the SIMPLE endoscopic classification of diminutive and small colorectal polyps. Endoscopy 2018; 50: 779-789

[10] Rees C], Rajasekhar PT, Wilson A et al. Narrow band imaging optical diagnosis of small colorectal polyps in routine clinical practice: the Detect Inspect Characterise Resect and Discard 2 (DISCARD 2) study. Gut 2017; 66: 887-895

[11] van de Wetering AJP, Meulen LWT, Bogie RMM et al. Optical diagnosis of diminutive polyps in the Dutch Bowel Cancer Screening Program: Are we ready to start? Endosc Int Open 2020; 08: E257-E265

[12] Open El. Uptake and barriers for implementation of the resect and discard strategy. 2020; 08: E684-E692 
[13] Rex DK, Patel N], Vemulapalli KC. A survey of patient acceptance of Resect and Discard for diminutive polyps. Gastrointest Endosc 2015; 82: 376-380.e1

[14] Bisschops R, Dekker E, East JE et al. European Society of Gastrointestinal Endoscopy (ESGE) curricula development for postgraduate training in advanced endoscopic procedures: rationale and methodology. Endoscopy 2019; 51: 976-979

[15] Esteva A, Kuprel B, Novoa RA et al. Dermatologist-level classification of skin cancer with deep neural networks. Nature 2017; 542: 115-118

[16] Byrne MF, Chapados N, Soudan F et al. Real-time differentiation of adenomatous and hyperplastic diminutive colorectal polyps during analysis of unaltered videos of standard colonoscopy using a deep learning model. Gut 2019; 68: 94-100

[17] Bernal J, Tajkbaksh N, Sanchez FJ et al. Comparative validation of polyp detection methods in video colonoscopy: Results From the MICCAI 2015 Endoscopic Vision Challenge. IEEE Trans Med Imaging 2017; 36: 1231-1249
[18] Chen P-J, Lin M-C, Lai M-J et al. Accurate classification of diminutive colorectal polyps using computer-aided analysis. Gastroenterology 2018; 154: 568-575

[19] Urban G, Tripathi P, Alkayali T et al. Deep learning localizes and identifies polyps in real time with $96 \%$ accuracy in screening colonoscopy. Gastroenterology 2018; 155: 1069-1078.e8

[20] Kamiński MF, Hassan C, Bisschops R et al. Advanced imaging for detection and differentiation of colorectal neoplasia: European Society of Gastrointestinal Endoscopy (ESGE) Guideline. Endoscopy 2014; 46: 435-449

[21] Byrne MF, Chapados N, Soudan F et al. Real-time differentiation of adenomatous and hyperplastic diminutive colorectal polyps during analysis of unaltered videos of standard colonoscopy using a deep learning model. Gut 2019; 68: 94-100

[22] Kudo S-E, Misawa M, Mori Y et al. Artificial intelligence-assisted system improves endoscopic identification of colorectal neoplasms. Clin Gastroenterol Hepatol; 2020: (In press) https://linkinghub.elsevier. com/retrieve/pii/S1542356519309978 